was 6/12. Slit-lamp examination at this time showed punctate deposits of ferrous pigment on the posterior capsule. On May 15, discission of the post. capsule was performed with a Ziegler knife and a good gap obtained. The patient was last seen on July 6 . Vision was then correctable to $6 / 12$. The visual field was contracted down to $45^{\circ}$ on the temporal side and to $20^{\circ}$ on the nasal side. Fundus details were seen but hazily. The patient returned to duty in Medical Category A.4.

\title{
THE EFFECT OF OPERATIVE ALTERATIONS IN THE HEIGHT OF THE EXTERNAL RECTUS INSERTION*
}

BY

\section{John Foster and E. C. Pemberton}

\section{LEEDS}

IT will be observed from the literature that operative correction of the vertical element of convergent concomitant squint is more readily undertaken in America than in England.

Such correction is usually obtained in America by operating on the superior or inferior recti and obliques, and may precede operation on the horizontal muscles.

We suspect that the wide use of the cover test of Duane which is more informative than the major amblyoscope in cardinal positions above and below the horizontal, may be responsible for this.

The writers venture to doubt if there is in Great Britain even a single set of the large ( $37 \mathrm{~mm}$.) square prisms which facilitate quantitative application of Duane's test. These remarks in no way decry the accuracy of the synoptophore as an indicator of the degree of fusion or the horizontal or vertical angles near the horizontal plane and with which all measurements in this paper were made. Where marked alterations in the vertical angle take place during horizontal movement, an operation on a vertically acting rectus or an oblique muscle is unavoidable, but where this angle is small and constant, attempts have been made by certain European surgeons to correct this vertical element by raising or lowering the external rectus while advancing or resecting it.

Differing opinions have been expressed verbally to me by colleagues, on the effect of deliberately raising the insertion. Some believe it elevates the eye, others that it depresses it.

Ronne (Acta Ophthal.23. I.fasc.1, p. 48) has expressed the third

* Received for publication, November 28, 1945. 
view that if the muscle is raised without raising the conjunctiva there is no vertical effect at all.

In an attempt to settle this problem, as my own clinical results had been uncertain (Trans. Ophthal. Soc. U.K., 1942 LXII. 320) the external rectus was attached to the upper part of the limbus under surface cocaine anaesthesia in two eyes prior to excision, and appeared to depress the eye.

\section{Qualitative effect}

Careful measurements in 26 squint cases operated on by myself or assistants since that date, and measured by E. C. Pemberton, Senior Orthoptist at the Leeds General Infirmary, show that the converse is the case. Elevation of the insertion of the external rectus elevates the eye, lowering it depresses the eye. The same rule applies to two cases where the internal rectus insertion was displaced vertically.

Theoretically one would expect cyclophoria to follow, and this did in fact occur in two cases, though without serious effect.

\section{Quantitative effect}

The external rectus in an eye with convergent squint varies so greatly in breadth and thickness, that one would not expect the same elevation of the globe from an identical procedure in each case. The figures given below are only averages, but in practice have proved consistent enough to be of help in making a decision clinically. It would appear that marked shortening of the muscle increases the vertical effect.

External rectus.

Whole width raising with resection and advancement $=11 \cdot 2 \Delta$

Whole width raising with resection alone $=7 \Delta$

Half width raising with resection and advancement $=9 \triangle$

Half width raising with resection alone: $=5.4 \Delta$

Internal rectus.

Half width raising $=2.75 \triangle$

In the tabulated results which follow, the horizontal error is quoted in degrees, and the vertical in prism dioptres, according to the usual convention for synoptophore measurements. The measurements were taken (a) pre-operatively and (b) one week $(c)$ one month post-operatively.

In one case owing to a misunderstanding, the muscle was raised instead of lowered, thus aggravating the error to an extent consistent with the above findings. 
Operations Involving Raising/Lowering of External Rectus for Correction $\stackrel{\vec{F}}{\vec{\rho}}$ of Hypertropia

N.B.-Horizontal error in Degrees; Vertical in Prism Dioptres

\begin{tabular}{|c|c|c|c|c|c|c|c|}
\hline & Case & No. & Pre-op. Dev. $\Delta$ & Operation & \begin{tabular}{|c|} 
Post-op. \\
Dev. $\triangle$ \\
1 week post-op.
\end{tabular} & $\begin{array}{c}\text { Final Dev. } \triangle \\
1 \text { month later }\end{array}$ & $\frac{\mathrm{O}}{\mathrm{O}} \operatorname{Red}{ }^{\mathrm{R}} \stackrel{\mathrm{O}}{\mathrm{O}}$ \\
\hline 1 & E. S. & 30179 & $+20^{\circ} \mathrm{R} / \mathrm{L} 5 \triangle$ & $\begin{array}{l}\text { R.E. Recession } 5 \mathrm{~mm} . \text {., } \\
\text { Resection } 5 \mathrm{~mm} \text {., with } \\
1 / 2 \text { breadth lowering }\end{array}$ & $-11 \mathrm{~L} / \mathrm{R} 1 \Delta$ & $\begin{array}{l}0^{\circ} \text { to }-3^{\circ} \\
\text { R/L } 2 \triangle \\
\text { S.B.V. }\end{array}$ & $\omega$ \\
\hline 2 & D. $\mathbf{w}$. & 37461 & $+34^{\circ} \mathrm{L} / \mathrm{R} 16 \triangle$ & $\begin{array}{l}\text { L.E. } 5: 7: 3 \text { with full } \\
\text { breadth lowering }\end{array}$ & $+7^{\circ} \mathrm{L} / \mathrm{R} 3 \triangle$ & \begin{tabular}{|} 
?dist. \\
Wing $\bar{c}$ gl. \\
Exo. 2 L/R $3 \triangle$ \\
S.B.V.
\end{tabular} & 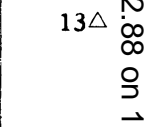 \\
\hline 3 & C. G. & $\begin{array}{l}5110 \\
\text { or } \\
6110\end{array}$ & $+22^{\circ} \mathrm{L} / \mathrm{R} 11 \triangle$ & $\begin{array}{l}\text { L.E. } 5: 7: 3 \text { with full } \\
\text { breadth lowering }\end{array}$ & $\begin{array}{c}+8^{\circ} L / R \\
1-2 \Delta\end{array}$ & $+8^{\circ} L / R i-2 \triangle$ & $10 \triangle \underset{\frac{\mathbb{D}}{\mathrm{O}}}{\frac{\mathrm{O}}{\mathrm{C}}}$ \\
\hline 4 & w. B. & 7000 & $\begin{array}{l}+15^{\circ} \mathrm{L} / \mathrm{R} \\
8-10 \triangle\end{array}$ & $\begin{array}{l}\text { L.E. } 5: 5 \text { with } 1 / 2 \\
\text { breadth lowering }\end{array}$ & $+9^{\circ} \mathrm{L} / \mathrm{R} 5 \Delta$ & $+8^{\circ} \mathrm{L} / \mathrm{R} 6 \triangle$ & $4 \triangle$ \\
\hline 5 & J. F. & 9482 & $+34^{\circ} \mathrm{L} / \mathrm{R}^{\prime} 11 \triangle$ & $\begin{array}{l}\text { R.E. } 5: 7: 4 \text { with } 1 / 2 \\
\text { breadth raising }\end{array}$ & $+7^{\circ} \mathrm{L} / \mathrm{R} 6 \triangle$ & $\begin{array}{c}+10^{\circ} \mathrm{L} / \mathrm{R} 4 \Delta \\
\text { R.E. extorsion } \\
7^{\circ} \text { (variable) }\end{array}$ & $7 \triangle$ \\
\hline 6 & I. W. & 1205 & $+28^{\circ} \mathrm{L} / \mathrm{R} 11 \triangle$ & $\begin{array}{l}\text { R.E. } 5: 5: 3 \text { with full } \\
\text { breadth raising }\end{array}$ & $\begin{array}{l}+9 \text { to } 11^{\circ} \\
\mathrm{L} / \mathrm{R} 1 \triangle\end{array}$ & $\begin{array}{c}+4^{\circ} \frac{\mathrm{O}}{\mathrm{O}} \text { nil } \\
\text { S.B.V. }\end{array}$ & $11 \triangle \frac{5}{\partial}$ \\
\hline 7 & G. W. & 8312 & $+23^{\circ} \mathrm{R} / \mathrm{L} 2 \triangle$ & $\begin{array}{l}\text { R.E. } 5: 7 \text { with } 1 / 2 \\
\text { breadth lowering }\end{array}$ & $\begin{array}{c}+5 \text { to } 9^{\circ} \mathrm{L} / \mathrm{R} \\
1 \triangle\end{array}$ & $+9^{\circ} \mathrm{L} / \mathrm{R} 5 \triangle$ & $7 \triangle$ \\
\hline 8 & J. M. & 10524 & $+27^{\circ} \mathrm{L} / \mathrm{R} 9 \triangle$ & $\begin{array}{l}\text { R.E. Adv. and Reces- } \\
\text { sion with full width } \\
\text { raising of R.E.R. }\end{array}$ & $+14^{\circ}$ R/L $5 \Delta$ & $+14^{\circ} \mathrm{R} / \mathrm{L} 5 \triangle$ & $\begin{array}{l}14 \triangle \text { and } \\
\text { over-章 } \\
\text { correct }\end{array}$ \\
\hline 9 & L. E. & 31146 & $\begin{array}{l}\text { Var. }+12^{\circ} \text { to } \\
+24^{\circ} \mathrm{L} / \mathrm{R} 15 \Delta\end{array}$ & $\begin{array}{l}\text { L.E. } 5: 5 \text { with full } \\
\text { width lowering }\end{array}$ & $+13^{\circ} \mathrm{L} / \mathrm{R} 12 \Delta$ & $+8^{\circ} L / R 8 \triangle$ & \\
\hline 10 & P. $\mathbf{H}$. & 2114 & $+16^{\circ} \mathrm{L} / \mathrm{R} 7 \triangle$ & $\begin{array}{l}\text { L.E. } 5: 5 \text { with full } \\
\text { width lowering }\end{array}$ & $-5^{\circ} \mathrm{L} / \mathrm{R} 1-3 \Delta$ & $0^{\circ} \frac{\mathrm{o}}{\mathrm{o}} \mathrm{nil}$ & \\
\hline $11^{\circ}$ & A. B. & 3.5055 & $+13^{\circ} \mathrm{R} / \mathrm{L} 6 \triangle$ & $\begin{array}{l}\text { R.E. Adv. + Rec. with } \\
1 / 2 \text { width lowering }\end{array}$ & $+7^{\circ} \mathrm{R} / \mathrm{L} 1 \triangle$ & $+7^{\circ} \mathrm{L} / \mathrm{R} 3 \triangle$ & $\begin{array}{r}9 \triangle \text { and } \overrightarrow{w_{i}} \\
\text { over-o } \\
\text { correctes }\end{array}$ \\
\hline 12 & R. F. & $\begin{array}{r}17487 \\
,\end{array}$ & $+28^{\circ} \mathrm{L} / \mathrm{R} 5 \triangle$ & $\begin{array}{l}\text { L.E. } 5: 10 \text { with } 1 / 2 \\
\text { width raising } \\
\text { L.E.R. (error) }\end{array}$ & $+1^{\circ} \mathrm{L} / \mathrm{R} 12^{\circ}$ & $+8^{\circ} \mathrm{L} / \mathrm{R} 9 \Delta$ & $\begin{array}{l}\mathrm{L} / \mathrm{R} \text { was. } \\
\text { increas } \\
\text { by } 4 \triangle \mathrm{RS}\end{array}$ \\
\hline 13 & J.P. & 17646 & $+42^{\circ} \mathrm{L} / \mathrm{R} 14 \triangle$ & $\begin{array}{l}\text { L.E. } 5: 9 \cdot 3 \text { with } 1 / 2 \\
\text { width lowering }\end{array}$ & $\begin{array}{l}+7^{\circ} \text { no } \frac{0}{0} \text { but } \\
\text { L. E. excycl. } 10^{\circ}\end{array}$ & $\begin{array}{l}+7^{\circ} \mathrm{R} / \mathrm{L} \cdot 1 \triangle \\
\mathrm{L} . \text { excycl. } 4^{\circ}\end{array}$ & $9-15$ त్ \\
\hline & L. A. & 11994 & $+22^{\circ} \mathrm{L} / \mathrm{R} .5 \triangle$ & $\begin{array}{l}\text { L.E. } 5: 6: 3 \text { with } 1 / 2 \\
\text { width lowering }\end{array}$ & $-1^{\circ}$ no $\frac{0}{0}$ & $-2^{\circ} \mathrm{R} / \mathrm{L} 9 \Delta$ & $\begin{array}{l}14 \Delta \text { (over } \\
\text { correctect }\end{array}$ \\
\hline 15 & P. G. & 37729 & $+25^{\circ} \mathrm{R} / \mathrm{L} 5 \triangle$ & $\begin{array}{l}\text { L.E. } 5: 6: 3 \text { with } 1 / 2 \\
\text { width raising }\end{array}$ & $+7^{\circ} L / R_{1} \triangle$ & Not seen since & \\
\hline
\end{tabular}


Height of the External Rectus Insertion

Operations Involving Raising/Lowering of External Rectus for Correction of Hypertropia-continued

N.B.-Horizontal error in Degrees ; Vertical in Prism Dioptres

\begin{tabular}{|c|c|c|c|c|c|c|}
\hline Case & No. & Pre-op. Dev $\triangle$ & Operation & $\begin{array}{c}\text { Post-op. } \\
\text { Dev. } \Delta \\
1 \text { week post-op. }\end{array}$ & $\begin{array}{l}\text { Final Dev. } \triangle \\
1 \text { month later }\end{array}$ & $\frac{\mathrm{O}}{\mathrm{O}}$ Reduc- \\
\hline I. $\mathbf{K}$. & 16880 & $\begin{array}{l}-13^{\circ} \text { to }-20^{\circ} \\
\quad \mathrm{L} / \mathrm{R} 7 \triangle\end{array}$ & $\begin{array}{l}\text { L.I.R. re-advancement } \\
\text { with } 1 / 2 \text { width lower- } \\
\text { ing of Int. Rect. }\end{array}$ & $\begin{array}{l}-3^{\circ} \text { to }-12^{\circ} \\
L / R 4 \Delta\end{array}$ & $\begin{array}{l}-8^{\circ} \text { to }-12^{\circ} \\
\text { L/R } 5 \triangle\end{array}$ & $2 \Delta$ \\
\hline L. M. & 13431 & $-28^{\circ} \mathrm{R} / \mathrm{L} 8 \Delta$ & $\begin{array}{l}\text { Tenot. L.E.R. } 4 \mathrm{~mm} \text {. } \\
\text { resect. L.I.R. } 3 \mathrm{~mm} \text {, } \\
\text { raising }\end{array}$ & $+4^{\circ} \mathrm{R} / \mathrm{L} 8 \Delta$ & $-2^{\circ} \mathrm{R} / \mathrm{L} 4-6 \Delta$ & $2-4 \Delta$ \\
\hline E. W. & 15535 & $+21^{\circ} \mathrm{L} / \mathrm{R} 6 \triangle$ & $\begin{array}{l}\text { R.E. } 5: 3 \text { with } 1 / 2 \\
\text { width raising }\end{array}$ & $\begin{array}{c}+10^{\circ} \text { to }+17^{\circ} \\
\mathrm{L} / \mathrm{R} 6 \Delta\end{array}$ & $+9^{\circ} \mathrm{L} / \mathrm{R} 6 \triangle$ & Nil effect \\
\hline G. P. & 7229 & $+35^{\circ} \mathrm{L} / \mathrm{R} 6-7 \Delta$ & $\begin{array}{l}\text { R.E. } 5: 10 \text { with } 1 / 2 \\
\text { width raise }\end{array}$ & $+13^{\circ} \mathrm{L} / \mathrm{R} 2 \triangle$ & $\begin{array}{c}+12^{\circ} \text { to }+16^{\circ} \\
\mathrm{L} / \mathrm{R} 3 \Delta\end{array}$ & $3-4 \Delta$ \\
\hline ) B. G. & 25393 & $+26^{\circ} \mathrm{R} / \mathrm{L} 9 \Delta$ & $\begin{array}{l}12 \mathrm{~mm} \text {. resect. L.E.R. } \\
\text { with } 1 / 2 \text { width raising } \\
\text { (had had previous bi- } \\
\text { lat. op.) }\end{array}$ & $+12^{\circ} \mathrm{R} / \mathrm{L} 5 \Delta$ & $+13^{\circ} \mathrm{R} / \mathrm{L}_{5} \triangle$ & $4 \Delta$ \\
\hline I I. E. & 6659 & $+20^{\circ} \mathrm{L} / \mathrm{R} 7 \Delta$ & $\begin{array}{l}\text { R.E. } 5: 8 \bar{c} 1 / 2 \text { width } \\
\text { raising }\end{array}$ & $\mid \begin{array}{r}0^{\circ} \mathrm{R} / \mathrm{L} 3 \triangle \mathrm{F} . \mathrm{R} . \\
\mathrm{L} / \mathrm{R} 2 \Delta \mathrm{F} . \mathrm{I} .\end{array}$ & $\begin{array}{c}+2^{\circ} \frac{\mathrm{O}}{\mathrm{O}} \mathrm{Nil} \\
\text { weak S.B.V. }\end{array}$ & $7 \triangle$ \\
\hline Mrs. & 29253 & $+40^{\circ} \mathrm{L} / \mathrm{R} 11 \triangle$ & $\begin{array}{l}\text { L.E. } 6: 10 \bar{c} 1 / 2 \text { width } \\
\text { lowering }\end{array}$ & $+6^{\circ} \mathrm{R} / \mathrm{L}_{1} \triangle$ & $+6^{\circ} \mathrm{L} / \mathrm{R} 4 \Delta$ & $\begin{array}{l}7 \triangle \text { under- } \\
\text { corrected }\end{array}$ \\
\hline 3 T. P. & 1134 & $+20^{\circ} \mathrm{R} / \mathrm{L} 3 \Delta$ & $\begin{array}{l}\text { L.E. } 4: 4 \bar{c} 1 / 4 \text { width } \\
\text { raise }\end{array}$ & $+10^{\circ} \mathrm{R} / \mathrm{L} 1 \triangle$ & $+3^{\circ} \frac{\mathrm{o}}{\mathrm{O}} \mathrm{Nil}$ & $3 \triangle$ \\
\hline 4 E. M. & 23016 & $+20^{\circ} \mathrm{L} / \mathrm{R} \cdot 7 \triangle$ & $\begin{array}{l}\text { R.E. } 5: 4 \bar{c} 1 / 2 \text { width } \\
\text { raise }\end{array}$ & $+2^{\circ} \mathrm{R} / \mathrm{L} 7 \triangle$ & $+2^{\circ} \mathrm{L} / \mathrm{R} 2 \Delta$ & $5 \triangle$ \\
\hline M. C. & 20144 & $+19^{\circ} \mathrm{L} / \mathrm{R} 3-5 \Delta$ & $\begin{array}{l}\text { R.E. } 5: 7 \bar{c} 1 / 2 \text { width } \\
\text { raise }\end{array}$ & $+10^{\circ} \mathrm{R} / \mathrm{L} 6 \triangle$ & $\begin{array}{l}+3^{\circ} \mathrm{R} / \mathrm{L} 2 \triangle \\
\text { occas. S.B.V. }\end{array}$ & $5-7 \Delta$ \\
\hline J. L. & 31058 & $+39^{\circ} \mathrm{L} / \mathrm{R} 34$ & $\begin{array}{l}\text { R.E. } 5: 10: 41 / 4 \text { width } \\
\text { raising }\end{array}$ & $-5 \mathrm{R} / \mathrm{L} 2-4 \Delta$ & $\begin{array}{l}+4^{\circ} \frac{\mathrm{o}}{\mathrm{O}} \mathrm{Nil} \\
\text { occas. S.B.V. }\end{array}$ & $3 \Delta$ \\
\hline
\end{tabular}

The figures in the column labelled " operation " represent the recession, resection, and advancement respectively, in millimetres. Cf. $5: 7: 3$ represents $5 \mathrm{~mm}$. recession, $7 \mathrm{~mm}$. reseçtion, $3 \mathrm{~mm}$. advancement.

Six Nos. 1, 2, 6, 21, 26 of these cases were having orthoptic treatment, and it was hoped that alteration of the vertical at the same time as that of the horizontal element, might lead to single binocular vision. In three cases this was achieved within a month, and in two others the continuing improvement in the squint leads 
the orthoptic department to think that it will still occur. In one other case there is no possibility of it occurring. Single binocular vision also occurred unexpectedly in two cosmetic cases after operation.

As the conjunctiva was completely detached from the muscle before advancement or resection, and carefully, replaced in its original position afterwards, it is difficult to believe it has any effect on the position of the eye in our cases.

\title{
Summary
}

The results of raising or lowering the external rectus insertion on twenty-sif cases of concomitant squint suggest that :-

(1) There is some justification for this when the vertical error is constant, and less than $11^{\Delta}$. at all horizontal angles.

(2) The eye moves in the direction in which the insertion is moved.

- (3) This movement is independent of the position of the conjunctiva.

\section{MENINGIOMA OF THE TUBERCULUM SELLAE WITH BI-TEMPORAL HEMIANOPIA*}

BY

\author{
W. R. MatheWSON
}

EDINBURGH

IN October, 1942, a woman, aged 61 years, complained of inability to see with the left eye, duration three months, but vision was R.E. 6/18 and L.E. 6/18 and was corrected to R.E. 6/6/pt. and L.E. $6 / 6 / \mathrm{pt}$., in spite of early lens opacities. Later her physician, Dr. Ivor Menzies, reported the general health satisfactory and the Wassermann reaction negative. Dr. A. H. H. Sinclair kindly saw her later in consultation, confirming the fields and adding further points, whilst Dr. Malcolm Farquharson reported Ear, Nose and Throat examination negative, as likewise were X-rays, taken by Drs. King and Allen.

Mr. Norman Dott operated on January 12, 1944, after further investigations including lumbar puncture and electro-encephalogram.

In May, 1943, vision with glasses was R.E. 6/18, L.E. 6/12, and though field defects were instinctively suspected, the diminution was too largely ascribed to lens changes, and field examination deferred, though partly on account of her temperament, which it was feared would render field examination long and difficult; a

\footnotetext{
- Received for publication, May 31, 1945.
} 